\title{
The effects of plastic waves on the numerical convergence of the viscous-plastic and elastic-viscous-plastic sea-ice models
}

\author{
James Williams* and L. Bruno Tremblay \\ Department of Atmospheric and Oceanic Sciences, McGill University, 805 Sherbrooke \\ Ouest, Montréal, QC, Canada H3A 2K6 \\ Jean-François Lemieux \\ Recherche en Prévision Numérique environnementale, Environnement et Changement \\ Climatique Canada, 2121 route Transcanadienne, Dorval, QC H9P 1J3, Canada
}

\begin{abstract}
The plastic wave speed is derived from the linearized 1-D version of the widely used viscous-plastic (VP) and elastic-viscous-plastic (EVP) sea-ice models. CourantFriedrichs-Lewy (CFL) conditions are derived using the propagation speed of the wave. 1-D numerical experiments of the VP, EVP and EVP* models successfully recreate a reference solution when the CFL conditions are satisfied, in agreement with the theory presented. The IMplicit-EXplicit (IMEX) method is shown to effectively alleviate the plastic wave CFL constraint on the timestep in the implicitly solved VP model in both 1-D and 2-D. In 2-D, the EVP and EVP* models show first order error in the simulated velocity field when the plastic wave is not resolved. EVP simulations are performed with various advective timestep, number of subcycles, and elastic-wave damping timescales. It is found that increasing the number of subcycles beyond that needed to resolve the elastic wave does not improve the quality of the solution. It is found that reducing the elastic wave damping timescale reduces the spatial extent of first order errors cause by the unresolved plastic wave. Reducing the advective timestep so that the plastic wave is resolved also reduces the velocity error in
\end{abstract}

*Corresponding author, e-mail: james.williams@mail.mcgill.ca

Preprint submitted to Journal of $E^{A} T_{E} X$ Templates

March 24, 2017

(C) 2017. This manuscript version is made available under the Elsevier user license http://www.elsevier.com/open-access/userlicense/1.0/ 
terms of magnitude and spatial extent. However, the parameter set required for convergence to within the error bars of satellite (RGPS) deformation fields is impractical for use in climate model simulations. The behavior of the EVP* method is analogous to that of the EVP method except that it is not possible to reduce the damping timescale with $\alpha=\beta$.

Keywords: Sea ice, Arctic, Numerical modeling, Rheology

\section{Introduction}

There are two types of waves in sea ice: elastic waves which propagate through sea ice as small reversible deformations and larger amplitude plastic waves which result in irreversible changes to the structure of the ice. Elastic 5 wave speeds of several km/s have been measured in the Arctic pack ice [e.g. 1, 2, These waves are well understood in terms of linear elastic theory with the wave speed depending on the Young's modulus and density of sea ice. However, due to their large speed these waves impose a very strict constraint on the time step of the numerical solution. In order to model the reversible nature of the elastic deformation, the deformation history of the ice must also be tracked [3]. Due to these restrictions, elastic deformations are typically neglected in large-scale numerical models of sea ice, such as in the widely used Viscous-Plastic (VP) model of Hibler [4]. The speed of the plastic deformation wave (ridge formation) on the other hand depends on the sea-ice yield strength parameterization and the ice density. For typical model values, the plastic wave propagates orders of magnitude slower than the elastic wave, but still much faster $\left(\sim 26 \mathrm{~m} \mathrm{~s}^{-1}\right)$ than the velocity of the ice [5]. This wave, for instance, represents the propagation of convergent motion away from the initial point of failure and is therefore an inherent feature of the widely used VP and Elastic-Viscous-Plastic (EVP) sea-ice model. Since the speed of the plastic wave is larger than the sea-ice drift speed, it imposes a more stringent constraint on the maximum advective timestep for a given spatial grid in the VP model than the sea-ice drift speed. For instance, with a spatial resolution of $5 \mathrm{~km}$, it is necessary to have a timestep 
less than 192 seconds $\left(5000 \mathrm{~m} / 26 \mathrm{~m} \mathrm{~s}^{-1}\right)$ for the model to correctly resolve plastic waves. Current models used in the community typically do not respect this condition.

The VP sea-ice model of Hibler [4] was originally iterated point by point using the successive over relaxation (SOR) method to integrate the velocity fields in time. The implicit treatment of the momentum equation was chosen due to the impractically small timestep required for an explicit time marching scheme to be stable. Then, after the momentum balance was solved for a given timestep, an explicit advection scheme was used to update the local thickness and concentration fields. Later, Zhang and Hibler [6] proposed a line successive over relaxation (LSOR) method to improve the numerical efficiency of the model for a given error tolerance. The LSOR method effectively updates the momentum equation row by row (or column by column) rather than point by point as in the SOR.

With the motivation to run the sea-ice model in a parallel computing environment, Hunke and Dukowicz [7] proposed to add a time dependence to the stress tensor. This explicit time dependence introduces an artificial elastic wave to the solution. The time dependent stress tensor and momentum equations were then sub-cycled with fixed viscosity coefficients at a subcycling timestep much shorter than the advective timestep in order to maintain numerical stability and sufficiently damp the elastic wave. This new formulation was termed ${ }_{45}$ the Elastic-Viscous-Plastic (EVP) model. The authors argued that the resulting stability conditions of the EVP model allow for an explicit timestepping scheme with a larger timestep. The EVP approach was then modified to allow the viscous coefficients to change during the sub-cycling procedure. This allowed for the state of stress of all points in the model domain to lie on or within the elliptical yield curve [8].

Building on previous implicit approaches used by Hibler [4] and Zhang and Hibler [6], Lemieux et al. 9] opted to solve the linearized momentum equation at each Picard iteration using a preconditioned Generalized Minimal RESidual (GMRES) method. Later, Lemieux et al. [10] proposed the Jacobian-Free 
Newton-Krylov (JFNK) method in order to accelerate the numerical convergence rate of the fully non-linear set of equations. Following this, Lemieux et al. 11] compared the EVP solution to an implicit (JFNK) reference solution computed to machine precision with a one second timestep. The authors concluded that the EVP method does not converge to the same solution as the VP model and that first order errors found in the velocity field are due to residual artificial elastic waves which are not fully damped on the advective timescale. Furthermore, grid cell noise in the deformation fields of the EVP solution was noted.

Lemieux et al. 11] also introduced the EVP* method, consisting of treating the original EVP time derivative as an unsteady correction term which should be iterated until it is near zero, in order to recover the original VP momentum equation. A second time derivative term is added to the momentum equation to represent the local time derivative. The time discretization of the $\mathrm{EVP}^{*}$ method and the implicit scheme can then be made to be identical and therefore the $\mathrm{EVP}^{*}$ method and implicit models should converge to the same solution to within machine precision. This led Lemieux et al. [11 to use the EVP* method as a tool to validate their numerical implementation of the EVP method. However, the authors also noted that the $\mathrm{EVP}^{*}$ method only converged to the implicit solution when the ice strength was reduced by an order of magnitude. Later, 75 Bouillon et al. [12] reinterpreted the EVP* method (called the modified EVP in their work) as an iterative solver and found that grid-cell noise in the seaice deformation fields (similar to those shown by Lemieux et al. [1] with the original EVP method) could be removed in the EVP* scheme. Kimmritz et al. 13 derived a numerical stability condition for the EVP* method (called interchangeably the EVP* or the modified EVP in their work). They showed that if the stability condition respected and a large number of subcycles are performed, the $\mathrm{EVP}^{*}$ method effectively does converge to within machine precision of the original viscous-plastic solution at spatial resolutions coarser than 0.1 degree. They report that fully converged solutions were unobtainable at resolutions of ${ }_{85} 0.1$ and 0.05 degrees. Furthermore, Kimmritz et al. [14] have introduced the 
adaptive EVP version of the EVP* method which allows for numerical parameters to vary spatially in order to relax stability constraints locally.

Hutchings et al. 15] showed that erroneous grid-point noise in the simulated sea-ice thickness field arises due to the split in time coupling of the momentum 90 and continuity equations. The authors proposed the Strength-Implicit method where the ice strength was updated in an implicit Picard solver in order to eliminate the noise in the solution. Their method is similar to the ImplicitExplicit (IMEX) method proposed by Lemieux et al. [16] in order to remove a similar grid cell noise. Both methods allow for the ice mass to be redistributed multiple times during the advective timestep which reduces the noise in the solution. Lipscomb et al. 17] documented noise in their multi-thickness category simulations and attributed this noise to the same grid point instability. They effectively eliminate the noise in their model by experimenting with different formulations of the ice thickness distribution and modifying the ridging scheme parameterization so that the ice strength changes more smoothly between time levels. This effectively changed the model physics rather than handling the numerical instability.

In this paper, we derive the speed of the plastic wave -following Gray [5] - and document the effects of integrating the governing equations using a timestep that ${ }_{105}$ is too large to resolve the plastic wave in 1-D and 2-D versions of the VP, EVP and $\mathrm{EVP}^{*}$ models. In this way, we present a theoretical explanation for the noise noted by Hutchings et al. [15], Lipscomb et al. [17] and Lemieux et al. [16. Using the derived Courant-Friedrichs-Lewy (CFL) condition, we show what advective timestep is required to resolve the plastic wave in numerical simulations using the VP, EVP or EVP* models. We show that 1-D numerical simulations largely match the theory presented in the three different models, and that the plastic wave must be well resolved in order to recreate a reference solution. We find that using the JFNK+IMEX method allows the model to be run with an arbitrarily long timestep, without the noise associated with failing to resolve the plastic wave. Furthermore, we show that applying a CFL condition of the elastic sound wave in the viscous (compressible) regime of the EVP model yields a stability 
condition analogous to the one derived by Kimmritz et al. [13] for the EVP* model. In 2-D we find that the EVP and EVP* models are more sensitive to the resolution of the plastic wave than the JFNK method. We find that reducing the damping timescale of the elastic wave provides significant improvement to the approximate solution found using the EVP method although a significantly greater number of subcycles is required. We show that the EVP and EVP* methods show similar error when the relevant stability conditions are satisfied. We discuss the implications of numerical errors in climate model simulations in the context with comparisons to satellite observations.

The paper is structured as follows. We first present the VP and EVP formulations of the model in Section 2. Section 3 is devoted to a theoretical understanding of the governing equations of the 1-D versions of the VP and EVP models. In section 4, we present results from 1-D and 2-D numerical simulations using the VP, EVP and EVP* models. In section 5, we discuss and summarize the results presented here.

\section{Model Description}

\subsection{2-D Viscous-Plastic Model}

The temporal evolution of the sea-ice velocity, thickness and concentration fields are described by the laws of conservation of momentum and mass. The momentum equation is:

$$
\rho_{i} h \frac{\partial \mathbf{u}}{\partial t}+\rho_{i} h f \hat{\mathbf{z}} \times \mathbf{u}=\tau_{\mathbf{a}}-\tau_{\mathbf{w}}-\rho_{i} h g \nabla H_{d}+\nabla \cdot \sigma,
$$

where $\rho_{i}$ is the density of sea ice, $h$ is the grid cell mean sea-ice thickness, $\mathbf{u}$ is the 2-D horizontal sea-ice velocity vector, $f$ is the Coriolis parameter, $\tau_{\mathbf{a}}$ is the surface air stress, $\tau_{\mathbf{w}}$ is the stress imparted on the bottom of the ice by the ocean, $g$ is the gravitational acceleration, $H_{d}$ is the dynamic sea surface height and $\sigma$ is the vertically integrated 2-D internal ice stress tensor. Note that we have neglected the advection of momentum in Equ. 1. The values of physical parameters are defined in Table 1. 
The surface air and ocean stresses acting on the ice, $\tau_{\mathbf{a}}$ and $\tau_{\mathbf{w}}$ respectively, as:

$$
\zeta=\frac{P}{2 \Delta}
$$

where $\Delta=\sqrt{\left(\dot{\epsilon}_{11}+\dot{\epsilon}_{22}\right)^{2}+\left(\frac{\dot{\epsilon}_{11}-\dot{\epsilon}_{22}}{e}\right)^{2}+\left(\frac{2 \dot{\epsilon}_{12}}{e}\right)^{2}}$ and,

$$
\eta=\frac{\zeta}{e^{2}}
$$


In the limit where $\Delta$ goes to zero, $\zeta$ and $\eta$ tend to infinity. In this case we use

the regularization of $\zeta$ proposed by Lemieux and Tremblay [19]:

$$
\zeta=\zeta_{\max } \tanh \left(\frac{P}{2 \Delta \zeta_{\max }}\right)
$$

with $\zeta_{\max }=2.5 \times 10^{8} \mathrm{P}$. This capping effectively sets the viscosity for grid cells in the viscous regime of the model. Following Hibler [4, we parameterize the sea-ice strength as a function of $\mathrm{h}$ and grid cell sea-ice concentration, $\mathrm{A}$, as:

$$
P=P^{*} h \exp [-C(1-A)],
$$

for the computation of the viscous coefficients. Then we use the replacement pressure method to recompute the ice strength used in the stress term (Equ. 4 of the momentum equation (Equ. 1) as $P=P_{R}=2 \Delta \zeta$ [20]. Note that this implies that $P_{R}$ only differs from $\mathrm{P}$ as calculated from Equ. 9 in the viscous regime of the model i.e. when $\zeta=\zeta_{\max }$.

The two continuity equations defining the temporal evolution of $\mathrm{h}$ and $\mathrm{A}$ (in the absence of thermodynamic source terms) can be written as:

$$
\begin{aligned}
& \frac{\partial h}{\partial t}+\nabla \cdot(h \mathbf{u})=0, \\
& \frac{\partial A}{\partial t}+\nabla \cdot(A \mathbf{u})=0,
\end{aligned}
$$

Typically, first the momentum equation (Equ. 1) is solved, and then the two continuity equations are updated using the newly found velocity field. We use this approach in the following..

\subsection{2-D Elastic-Viscous-Plastic Model}

A simple scale analysis of the linearized momentum equations considering only the $\frac{\partial \mathbf{u}}{\partial t}$ term and the rheology term (with $\zeta=\zeta_{\max }$ ) shows that a time step on the order of 0.01 seconds must be used for an explicit numerical scheme on a $10 \mathrm{~km}$ spatial grid. In order to relax this constraint, Hunke and Dukowicz [7] proposed to add a time dependence to the stress tensor effectively introducing an artificial elastic wave to the solution. Equ. (5) then becomes:

$$
\dot{\epsilon}_{i j}=\frac{1}{E} \frac{\partial \sigma_{i j}}{\partial t}+\frac{1}{2 \eta} \sigma_{i j}+\left(\frac{\eta-\zeta}{4 \eta \zeta} \sigma_{k k}+\frac{P}{4 \zeta}\right) \delta_{i j},
$$


where the new parameter, E, is analogous to the Young's modulus in an elastic material. Note that the E used in the EVP method is intended to be orders of magnitude smaller than the observed Young's modulus of Arctic sea ice in order not to impose a strict condition on the subcycling timestep, yet to allow faster numerical convergence. Hunke [8] defines $E=\frac{\zeta}{T}$, where $T$ is the damping timescale of the elastic wave. Hunke 8 chooses to set $T=0.36 \Delta t$. We keep $T$ as a free parameter, defined as: $T=T_{0} \Delta t$. The new system of equations is now:

$$
\begin{gathered}
\rho_{i} h \frac{\partial \mathbf{u}}{\partial t}+\rho_{i} h f \hat{\mathbf{z}} \times \mathbf{u}=\tau_{\mathbf{a}}-\tau_{\mathbf{w}}-\rho_{i} h g \nabla H_{d}+\nabla \cdot \sigma, \\
\frac{\partial \sigma_{i j}}{\partial t}=E\left[\dot{\epsilon}_{i j}-\frac{1}{2 \eta} \sigma_{i j}-\left(\frac{\eta-\zeta}{4 \eta \zeta} \sigma_{k k}+\frac{P}{4 \zeta}\right) \delta_{i j}\right] \\
\frac{\partial h}{\partial t}+\nabla \cdot(h \mathbf{u})=0, \\
\frac{\partial A}{\partial t}+\nabla \cdot(A \mathbf{u})=0 .
\end{gathered}
$$

The EVP solution method then entails integrating explicitly the momentum equation and stress equation for a number of subcycles $(N)$ for each advective timestep. This yields an effective timestep of $\Delta t_{e}=\frac{\Delta t}{N}$ for Equ. 13 and Equ. (14). The continuity equations are then updated after the subcycling procedure. The underlying assumption behind the EVP method is that the elastic waves are sufficiently damped on the advective timescale, and the original VP solution is recovered.

\section{3. $E V P^{*}$ Method}

Lemieux et al. 11] proposed the modified EVP (or EVP*) by adding an implicit version of the sea-ice inertial term to the original EVP momentum equations [8]. The resulting momentum equation discretized in time is:

$$
\beta^{*} \frac{\left(\mathbf{u}^{k+1}-\mathbf{u}^{k}\right)}{\Delta t_{e}}+\rho_{i} h \frac{\left(\mathbf{u}^{k+1}-\mathbf{u}^{n}\right)}{\Delta t}+\rho_{i} h f \hat{\mathbf{z}} \times \mathbf{u}^{k}=\tau_{\mathbf{a}}-\tau_{\mathbf{w}}-\rho_{i} h g \nabla H_{d}+\nabla \cdot \sigma,
$$

where the superscript $\mathrm{k}$ refers to the subcycling iteration level and the super-

script $\mathrm{n}$ represents the advective time level. The above equation thus represents 
the update of the velocity field from the $k^{t h}$ to the $k^{t h}+1$ subcycle of the $n^{t h}+1$ model timestep. In this sense, when the subcycling procedure has fully converged, $\mathbf{u}^{n+1}=\mathbf{u}^{k+1}=\mathbf{u}^{k}$, Equ. (17) will be exactly the same as the timediscretized version of Equ. (1). $\beta^{*}$ is a free parameter typically defined as an artificial mass, $\rho_{i} h$ [11, 12, 13]. Note that for the EVP* method, $\Delta t_{e}$ is no longer restricted to be $\Delta t_{e}=\Delta t / N$, as it was in the original EVP method. The timestepping of the stress equation (Equ. 14) in the EVP* method is the same as that of the EVP method.

Bouillon et al. [12] reinterpreted the EVP* method as analog to a non-linear iterative solver. Kimmritz et al. [13] further analyzed a similar formulation of the EVP* method. The authors write the set of equations as:

$$
\begin{gathered}
\sigma^{k+1}=\sigma^{k}+\frac{1}{\alpha}\left(\frac{\zeta^{k}}{\partial u^{k}} \partial x-\sigma^{k}\right) \\
u^{k+1}=u^{k}+\frac{1}{\beta}\left(\frac{\Delta t}{\rho h} \frac{\partial \sigma^{k+1}}{\partial x}+\frac{\Delta t}{\rho h} \tau_{a}+u_{n}-u_{k}\right),
\end{gathered}
$$

where the subscript $\mathrm{n}$ refers to the previous time level, and $\alpha$ and $\beta$ are numerical parameters which can be set as $\alpha=\frac{2 T}{\Delta t_{e}}$ and $\beta=\frac{\Delta t}{\Delta t_{e}}$. Kimmritz et al. [13] ${ }_{225}$ argued that $\alpha$ should be set equal to $\beta$, which in the EVP* model corresponds to:

$$
\frac{2 T}{\Delta t_{e}}=\frac{\Delta t}{\Delta t_{e}}
$$

or simply $2 T=\Delta t$. We see that the choice of $\alpha=\beta$ effectively sets the damping time scale parameter $T_{0}$ as defined in the previous section, and then either $\alpha$ or $\beta$ can be used to set $\Delta t_{e}$ as a function of the advective timestep. Kimmritz et al. 13 provided a stability condition for the EVP* method based on $\alpha$ and $\beta$ which we will adhere to in the following.

\section{1-D Analysis and Solutions}

\subsection{Plastic Wave: VP}

Following Gray [5], we derive the speed of the plastic deformation wave in the 1-D version of the conservation laws:

$$
\rho_{i} h \frac{\partial u}{\partial t}=\rho_{a} C_{d a}\left|u_{a}\right| u_{a}-\rho_{w} C_{d w}\left|u_{w}-u\right|\left(u_{w}-u\right)+\frac{\partial \sigma_{11}}{\partial x},
$$




$$
\begin{aligned}
& \frac{\partial h}{\partial t}+\frac{\partial}{\partial x}(h u)=0, \\
& \frac{\partial A}{\partial t}+\frac{\partial}{\partial x}(A u)=0,
\end{aligned}
$$

where $v=0, \sigma_{12}=\sigma_{21}=\sigma_{22}=0, \frac{\partial}{\partial y}$ of all variables is set equal to 0 , and we have assumed no sea-surface tilt. Note that we have neglected the advection of momentum in Equ. (21) as is typically done in 2-D. The constitutive law Equ. (4) in 1-D can be written as:

$$
\sigma=\sigma_{11}=\left(1+e^{-2}\right) \zeta \frac{\partial u}{\partial x}-\frac{P}{2} .
$$

Substituting Equ. (6) into Equ. (24) we find that $\sigma$ depends spatially only on the ice strength $\mathrm{P}$ and the sign of $\frac{\partial u}{\partial x}$ as:

$$
\sigma=\frac{P}{2}\left(\sqrt{1+e^{-2}} \frac{\frac{\partial u}{\partial x}}{\left|\frac{\partial u}{\partial x}\right|}-1\right) .
$$

This means that the stress only depends on the ice strength, $\mathrm{P}$ :

$$
\sigma=\lambda P,
$$

where $\lambda=\frac{ \pm \sqrt{1+e^{-2}}-1}{2}$. Note that we have assumed that we are in the plastic regime of the model, and therefore the replacement pressure is not relevant here. The 1-D momentum equation is now:

$$
\rho_{i} h \frac{\partial u}{\partial t}=\rho_{a} C_{d a}\left|u_{a}\right| u_{a}-\rho_{w} C_{d w}\left|u_{w}-u\right|\left(u_{w}-u\right)+\lambda \frac{\partial P}{\partial x} .
$$

Substituting in for P from Equ. (9), we write:

$\rho_{i} h \frac{\partial u}{\partial t}=\rho_{a} C_{d a}\left|u_{a}\right| u_{a}-\rho_{w} C_{d w}\left|u_{w}-u\right|\left(u_{w}-u\right)+\lambda P^{*} e^{(-C[1-A])}\left(\frac{\partial h}{\partial x}+C h \frac{\partial A}{\partial x}\right)$.

Equ. (22), 23) and (28) make up a complete system with three equations and three unknowns $(\mathrm{u}, \mathrm{h}$, and $\mathrm{A})$. We linearize the set of equations around a rest state velocity field i.e. $u_{0}=0$ and a base state thickness and concentration fields $\left(h_{0}, A_{0}\right)$ which are assumed to be spatially uniform. We assume no forcing. We then impose a perturbation, $\left(u^{\prime}, h^{\prime}, A^{\prime}\right)$. Assuming the fields are sufficiently smooth, the perturbations will initially evolve according to:

$$
\rho_{i} h \frac{\partial u^{\prime}}{\partial t}=\lambda P^{*} e^{\left(-C\left[1-A_{0}\right]\right)}\left(\frac{\partial h^{\prime}}{\partial x}+C h_{0} \frac{\partial A^{\prime}}{\partial x}\right),
$$




$$
\begin{aligned}
& \frac{\partial h^{\prime}}{\partial t}+h_{0} \frac{\partial u^{\prime}}{\partial x}=0, \\
& \frac{\partial A^{\prime}}{\partial t}+A_{0} \frac{\partial u^{\prime}}{\partial x}=0 .
\end{aligned}
$$

Assuming a wave solution of the form $\left[u^{\prime}, h^{\prime}, A^{\prime}\right]=[\hat{u}, \hat{h}, \hat{A}] e^{i(k x-\omega t)}$ and simplifying, the set of equations becomes:

$$
\begin{gathered}
\rho_{i} h_{0} \omega \hat{u}+\lambda^{*}\left(k \hat{h}+k h_{0} C \hat{A}\right)=0, \\
-\omega \hat{h}+h_{0} k \hat{u}=0, \\
-\omega \hat{A}+A_{0} k \hat{u}=0,
\end{gathered}
$$

where $\lambda^{*}=\lambda P^{*} \exp \left[-C\left(1-A_{0}\right)\right]$. Solving for the phase speed of the traveling wave,$\frac{\omega}{k}$, we find:

$$
c_{p}=\frac{\omega}{k}=\sqrt{-\frac{\lambda^{*}}{\rho_{i}}\left(1+C A_{0}\right)} .
$$

For typical parameter values (Table 1) and assuming a base state sea-ice concentration near 1 (but such that $A_{0}+\hat{A}<1.0$ ), we find the linear wave speed of the perturbation to be $\sim 26 \mathrm{~m} \mathrm{~s}^{-1}$ when $\frac{\partial u}{\partial x}<0$. This represents the speed of propagation of a ridge forming when sea ice deforms in compression. Note that the dependence of the wave speed on the sign of $\frac{\partial u}{\partial x}$ comes from the definition of $\lambda$ above. When $\frac{\partial u}{\partial x}>0, \lambda$ changes signs, and thus the wave speed becomes imaginary implying no wave-like behavior (see Gray [5] for a discussion of the divergent case). Therefore we expect waves to only propagate following. From a ratio of the terms in Equ. (35), we see that the wave speed primarily depends on the $\frac{\lambda^{*}}{\rho_{i}} C A_{0}$. Note that the linear wave speed is not a function of the thickness of the ice (h) due to the choice of a linear dependence of $\mathrm{P}$ on $\mathrm{h}$. If a different parameterization for $\mathrm{P}$ was used, for example that of Hopkins [21], then the plastic wave speed would depend on $h$. The wave speed, however, depends strongly on $P^{*}$ through $\lambda^{*}$ and on C. As $A_{0}$ increases from $A_{0}=0.5$ to $A_{0}=0.99$, the wave speed rises rapidly from near $0 \mathrm{~m} \mathrm{~s}^{-1}$ 
to $26 \mathrm{~ms}^{-1}$ (Fig 1). This indicates that a plastic wave travels through the ice at a speed three orders of magnitude faster than typical sea-ice velocities in areas of high concentration when ridging takes place. In regions of lower sea-ice concentration, the wave propagates much slower, and thus does not constitute a significant CFL constraint on the model timestep. We stress that the simple linear wave speed derived here should be treated as a rudimentary estimate for the actual plastic wave speed in a numerical simulation. In practice, the plastic wave speed is likely modified by gradients in the thickness and concentration field, as well as the inherent non-linearity in the 2-D case. However, we show in section 4 that this plastic wave theory does provide some useful insight into the interpretation of numerical results.

\subsection{Viscous Regime: VP}

When $\zeta=\zeta_{\max }$ locally, we consider the ice located at that grid point to be in the viscous regime of the VP model. In this case we can no longer eliminate the strain rate dependence from the stress tensor, as we did in Equ. 25. Instead, following from Equ. (26), the stress tensor can be written as:

$$
\sigma=\left(1+e^{-2}\right) \zeta_{\max } \frac{\partial u}{\partial x}-\frac{P_{R}}{2}
$$

Substituting this viscous regime stress tensor into the 1-D momentum equation (Equ. 21), and applying the replacement pressure definition, we find:

$\rho_{i} h \frac{\partial u}{\partial t}=\rho_{a} C_{d a}\left|u_{a}\right| u_{a}-\rho_{w} C_{d w}\left|u_{w}-u_{i}\right|\left(u_{w}-u_{i}\right)+\left(1+e^{-2}\right) \zeta_{\max } \frac{\partial^{2} u}{\partial x^{2}}-\zeta_{\max } \frac{\partial^{2} u}{\partial x^{2}}$.

From the above equation, we see that the $\zeta_{\max }$ terms in the viscous regime acts as a diffusion operator, and will not support a wave-like solution. We highlight this here because we will see in section 3.4 below that it is this term which is modified in EVP formulation. We also see from Equ. (37), that the use of the replacement pressure effectively reduces the diffusion in the viscous regime in convergent flow. 


\subsection{Plastic Wave: EVP}

In 1-D, we write the EVP system of equations by adding the elastic time derivative to the 1-D stress tensor (Equ. 24):

$$
\rho_{i} h \frac{\partial u}{\partial t}=\rho_{a} C_{d a}\left|u_{a}\right| u_{a}-\rho_{w} C_{d w}\left|u_{w}-u\right|\left(u_{w}-u\right)+\frac{\partial \sigma}{\partial x},
$$

305

$$
\begin{aligned}
\frac{\partial \sigma}{\partial t}=E\left(\frac{\partial u}{\partial x}-\frac{\sigma}{\left(1+e^{-2}\right) \zeta}\right. & \left.-\frac{P}{2\left(1+e^{-2}\right) \zeta}\right), \\
\frac{\partial h}{\partial t}+\frac{\partial}{\partial x}(h u) & =0 \\
\frac{\partial A}{\partial t}+\frac{\partial}{\partial x}(A u) & =0 .
\end{aligned}
$$

The momentum equation and continuity equations remain unchanged when compared to the VP 1-D system, but the stress tensor now depends on time in a manner similar to Equ. (14). Substituting $E=\zeta / T$ (where $T=T_{0} \Delta t$ is the damping time scale of the elastic wave [8]) into Equ. [39, we write:

$$
\frac{\partial \sigma}{\partial t}=\frac{\zeta}{T} \frac{\partial u}{\partial x}-\frac{\sigma}{\left(1+e^{-2}\right) T}-\frac{P}{2\left(1+e^{-2}\right) T} .
$$

Substituting for $\zeta$ and again assuming a convergent ice velocity field (which implies no replacement pressure), we write:

$$
\begin{gathered}
\frac{\partial \sigma}{\partial t}=-\frac{1}{\left(1+e^{-2}\right) T}\left[\sigma+\frac{1+\sqrt{1+e^{-2}}}{2} P\right], \\
\frac{\partial \sigma}{\partial t}=-\frac{1}{\left(1+e^{-2}\right) T}[\sigma-\lambda P] .
\end{gathered}
$$

The system of equations is now one way coupled. That is $u$ depends on $\sigma$ during the subcycling timestep, but $\sigma$ does only depend on $u$ through the redistribution of mass at the advective timestep. In fact, Equ. 44 can be solved analytically as:

$$
\sigma^{n}=\lambda P+\left(\sigma^{n-1}-\lambda P\right) e^{\frac{-t}{\left(1+e^{-2}\right) T}}
$$

where $t$ is the time during a given advective timestep and superscript $n$ refers to the advective time level (recall that $\lambda=\frac{-\sqrt{1+e^{-2}}-1}{2}$, see section 3.1). We 
320 stress tensor as for the VP case (see Equ. 26). That is, in the 1-D strengthexplicit formulation, the plastic wave in the EVP system will be identical to that in the VP system if the elastic wave is sufficiently damped during the subcycling procedure. On the advective timestep, h, A and therefore $\mathrm{P}$ will be updated to 325 be in line with the new velocity field. It is at this time that the plastic wave can propagate in the model. In practical applications, the elastic wave will likely not be fully damped at the advective timestep. This implies that any residual velocity gradients resulting from the incompletely damped elastic wave will alter the numerical solution and potentially trigger more plastic waves. We seek to further understand the interaction between incompletely damped elastic waves and plastic waves through numerical simulation in the following section. Note that the loss of dependence on the strain rate in Equ. 45 implies there are no waves propagating during the subcycling procedure. This is not the case in the 2-D system, where the strain rate dependence of $\zeta$ does not cancel when it is inserted into the stress tensor. Similarly, in the EVP* method $\mathrm{h}$ and A are held constant during the subcycling procedure. Therefore, the propagation of plastic deformation in the $\mathrm{EVP}^{*}$ method will also propagate at the advective timestep as in the EVP method.

\subsection{Viscous Regime and Elastic Wave: EVP}

The viscous coefficients are effectively constant during the subcycling procedure in the viscous regime of the EVP model. Assuming no spatial gradient in $\mathrm{h}$ or A, the original analysis performed in Hunke and Dukowicz [7] applies in these regions. The elastic wave speed is given by:

$$
c_{E}=\sqrt{\frac{E}{\rho h}},
$$

or after substituting in for $E=\frac{\zeta}{T}$ as defined in Hunke [8]:

$$
c_{E}=\sqrt{\frac{\zeta_{\max }}{\rho h T}} .
$$


$\left.\zeta_{\max }=2.5 \times 10^{8} \mathrm{P}\right)$ a timestep of several hours $\left(\sim 10^{4} \mathrm{~s}\right)$ is required to keep $\mathrm{E}$ in line with the values observed by Weeks and Assur [22] i.e. $E=1.7-9.1 G P a$. Using a smaller $\Delta t$, as is typically done in current climate model simulations, implies that the artificial elastic waves are in fact traveling faster than the elastic wave during the subcycling procedure because the plastic wave only propagates at the advective time level.

Forming a CFL condition for resolving the elastic wave yields:

$$
\sqrt{\frac{\zeta_{\max }}{\rho h T}} \Delta t_{e}<\Delta x .
$$

For the EVP method: $\Delta t_{e}=\frac{\Delta t}{N}$. Therefore we can rewrite the necessary condition for stability for the EVP method as a function of $\Delta t, \Delta x$ and $N$ as:

$$
\sqrt{\frac{\zeta_{\max }}{\rho h T_{0}}} \frac{\sqrt{\Delta t}}{N \Delta x}<1
$$

Adhering to this condition does not directly imply that the EVP solution will converge to the VP solution, but rather that the elastic wave will be resolved on the model grid. The damping timescale of the elastic wave is proportional to the $T_{0}$. 8 . This implies that both the elastic wave speed and its damping depend on the parameter $T_{0}$. Increasing $T_{0}$ results in a slower elastic wave which makes it easier to resolve according to the CFL condition; however it also results in a reduced decay rate of the elastic wave (see Equ. 45). We note that the theoretical elastic wave speed discussed here should be treated as an estimate of the actual speed of the waves present in numerical models, due to the inherent non-linearity in the stress tensor which in general will alter the speed. We test this theory experimentally in the following section.

Equ. (48) is identical to a within a factor $\pi$ of the stability condition presented by Kimmritz et al. 13. (Equ. 15 therein) for the EVP* method - using a different mathematical methodology. The differing constant comes from the fact 
the system, rather than using the wave speed (i.e. dividing by the wave number) to form a CFL condition as we have done above. This confirms that, in theory, a similar stability condition applies directly to the EVP system of equations as opposed to only the EVP* system of equations and offers an additional physical 375 context for why such stability conditions arise.

\section{Model Results and Analysis}

\subsection{1-D Simulations}

In one dimension, sea ice moving against a coastline will form a ridge. The ridged ice will extend offshore at an initial speed of tens of meters per second (see section 3.1). In order to resolve the propagation of this wave on the model grid, it is necessary to sample the wave sufficiently in accordance with the CFL criterion.

In a model with a first order advection scheme, this criterion is $c_{p}<\frac{\Delta x}{\Delta t}$ where $\Delta x$ and $\Delta t$ are the model grid spacing and advective timestep respectively. This condition effectively insures that the mass redistribution is properly resolved by the model advection scheme. Note that all simulations presented here use a simple 1st order upstream advection scheme. It is necessary to respect the CFL conditions of all processes within the model regardless of the numerical approach used to march the system forward in time.

To demonstrate this, we run a 1-D VP model described in Lemieux et al. [16] that is solved numerically using either the JFNK, EVP or, EVP* method. For all simulations, the wind forcing is constant in space and time with a speed $10 \mathrm{~ms}^{-1}$ in the positive $\mathrm{x}$ direction. The model domain is $2000 \mathrm{~km}$ in length with walls at both ends of the domain $(u=0 \mathrm{~m} / \mathrm{s})$. The initial conditions for the ice thickness, concentration and velocity are $1 \mathrm{~m}, 100 \%$ and $0 \mathrm{~m} / \mathrm{s}$ respectively from $1000 \mathrm{~km}-2000 \mathrm{~km}$. From $0-1000 \mathrm{~km}$ there is no ice, i.e. thickness and concentration are both zero. The spatial resolution of the model is $5 \mathrm{~km}$. This implies that the timestep must be smaller than $192 \mathrm{~s}$ according to the CFL criterion for the plastic wave. We first compute a reference solution to machine 
precision using the JFNK solver with $\Delta t=1 \mathrm{~s}$ to document the error for each solver (JFNK, EVP, EVP*).

We see that a grid cell instability develops when the timestep is 300 seconds or larger in simulations solved implicitly using JFNK (Fig. 2A). After forcing the model for one day, and $\Delta t=120 \mathrm{~s}$ (blue curve, directly underneath the black curve) remain in line with the reference solution, while the other simulations exhibit grid cell noise in the region of convergence resulting in first-order errors. This is evidence that the CFL condition derived for the linearized system is relevant to the full nonlinear system of equations. Note that the reference solution is indistinguishable from the the simulation with $\Delta t=30$ s (black curve) and is therefore not shown separately. This result is analogous to that presented in Lemieux et al. [16] however we provide here a theoretical understanding for why the instability develops based on the propagation of the plastic wave.

We find that using the IMEX method with the implicit solver (JFNK), as described in Lemieux et al. [16, effectively resolves the plastic wave despite the fact that the CFL condition applicable to the fully explicit advection scheme is not met (Fig 2B). Note that the divergence for all advective timesteps are indistinguishable from the reference solution with IMEX. This is because the coupling of the momentum and continuity equations is no longer split in time, as the thickness and concentration evolve within the implicit solver loop. The proper resolution of the plastic wave using the IMEX method with a timestep larger than the one implied by the CFL condition is additional evidence that the difference in the simulated divergence field from the exact solution is directly related to the splitting in time of the momentum and continuity equations. The IMEX method increases the run time of the model by $5-10 \%$, due to the greater number of advection calls per model timestep in 1-D for a given timestep. However, this effect is outweighed by the fact that a longer timestep can be used while still resolving the plastic wave. The reduced total number of timesteps when using IMEX thus results in a numerically less expensive simulation, assuming a timestep longer than that given by the CFL condition is chosen.

Similar erroneous deformation fields are found when using the EVP method 
with various advective timesteps and 1200 subcycles (Fig 2C.) Note that the elastic wave is resolved for all timesteps shown according to Equ. (48). We see that noise develops in the EVP solution when using a 120 second advective timestep which was not present in the implicit solution when using the same length timestep (Fig 2C: blue curve). Note that increasing the number of subcycles to 5000 for the EVP simulation does not alleviate this error seen with a $120 \mathrm{~s}$ advective timestep (not shown). On the other hand, reducing the damping timescale of the elastic wave $\left(T_{0}\right)$ by an order of magnitude does effectively eliminate the observed noise (not shown). This indicates that increasing the damping of the elastic wave is one potential way to reduce model error for the EVP method which as of yet has not been directly discussed in the literature. Furthermore, this shows that this departure from the reference solution is not caused by the different treatment of the time discretization between the EVP method and the implicit method. This also highlights the fact that satisfying both the plastic and elastic wave CFL conditions does not guarantee convergence to the reference solution. In addition to satisfying these two conditions, one must also ensure that the elastic wave is sufficiently damped at the advective time level.

We see a similar effect resulting from the unresolved plastic wave when using the $\mathrm{EVP}^{*}$ method (Fig. 2D). For all EVP* simulations presented, we use the method as laid out by Kimmritz et al. [13] with $\alpha=\beta=2000$. This set of parameters satisfies the stability conditions presented in Kimmritz et al. [13] for all advective timesteps shown. Note that the number of subcycles required for the $\mathrm{EVP}^{*}$ method is dictated by the choice of $\alpha$ and $\beta$. For our choice of $\alpha=\beta=2000$ we must increase the number of subcycles to several times $\alpha^{-1}+\beta^{-1}$; we choose 4000 subcycles 13 . Again, grid cell noise develops when the plastic wave is not resolved in a manner similar to the implicit solution (Fig 2D: green and red curve). We see that when the plastic wave is resolved the simulations match the reference solution (Fig. 2D: blue and black curve, note that the blue curve is directly under the black curve).

The error associated with not resolving the plastic wave and the elastic wave 
in the EVP model are spatially different. The error after 6 hours of forcing and a 600 second timestep with 430 subcycles $\left(N_{C F L} / 2\right)$ (Fig 3 red curve) and 860 $\left(N_{C F L}\right)$ subcycles (Fig 3 black curve) is largely due to the elastic wave not being resolved. Note that $N_{C F L}$ is the number of subcycles needed to satisfy Equ. (48). This is evidenced by the fact that the error is present over the entire domain (not only where deformation is occurring) and that it disappears once the elastic wave is resolved with 1760 subcycles $\left(2 N_{C F L}\right)$ (Fig 3 blue curve). The error that remains with 1760 subcycles (Fig 3 blue curve) is due to the fact that the plastic deformation wave is not resolved. We see that the error is found only in the region where large deformations are present. Note that in this simulation, ice was initialized over the entire domain, rather than only half of the domain as was the case in the previous simulations discussed. Analogous structure is seen with the EVP* method when stability condition of Kimmritz et al. 13 is not respected (not shown).

475 4.2. 2-D Simulations

\subsubsection{Model Setup and Reference Solution}

In this subsection, we evaluate the error associated with the JFNK, EVP and EVP $^{*}$ solvers using the sea-ice model described in Lemieux et al. [16]. To this end, we configure an idealized rectangular domain with land on all four sides. Ocean currents and thermodynamic ice growth or melt are set to zero for all time. The ice is forced with uniform eastward $5 \mathrm{~m} / \mathrm{s}$ winds. The spatially uniform initial conditions for ice thickness and concentration are $h=$ $1 m$ and $A=1$ respectively. The grid resolution is $10 \mathrm{~km}$. A reference solution is computed for this idealized domain using the JFNK method converged to machine precision with a $1 \mathrm{~s}$ advective timestep. After 6 hours of forcing, the velocity field is largely homogeneous with an eastward component of velocity of $7 \mathrm{~cm} / \mathrm{s}$ and a southward component of velocity of $0.5 \mathrm{~cm} / \mathrm{s}$. The ice thickness on the eastern edge of the domain has increased by $5 \mathrm{~cm}$. This reference solution is used in the next section to calculate the error associated with simulations computed with the JFNK, EVP and EVP* solvers. Note that according to the 
1-D plastic wave CFL condition we would need an advective timestep less than 385 seconds for a grid of $10 \mathrm{~km}$ spatial resolution.

\subsubsection{JFNK Solver}

The magnitude of the errors in the JFNK simulation with $\Delta t=600 \mathrm{~s}$ is

\subsubsection{EVP Solver}

First we test the EVP method using a 600 second advective timestep, typical of what is used in high-resolution operational forecast models. With 1200 subcycles and a damping timescale $T_{0}=0.36$ we see significant error in the 

Error greater than $1 \mathrm{~cm} / \mathrm{s}$ is present primarily near domain boundaries where deformation is taking place (Fig 5 top row). Note that in this simulation the elastic wave is resolved according to Equ. 48, while the plastic wave is not resolved. We found that increasing the number of subcycles as high as 20000 compared to the reference solution (not shown). The number of subcycles used in the simulation shown here are similar to the maximum number of subcycles used in the simulations presented in Lemieux et al. 11. We reproduce these results in order to create an error baseline associated with these typical paramWe use an idealized domain to confirm that the results of Lemieux et al. 11] are not associated with the open boundary conditions used in their simulations. Furthermore, we now interpret these results using the wave theory developed above in order to understand the root cause of the error.

We have shown in our analysis of the 1-D system that the elastic wave damping timescale $T_{0}$ is important for both the decay rate of the elastic wave and also for the propagation speed of the elastic wave. Thus we test the sensitivity of the model error to the value of the elastic wave damping timescale $T_{0}$. We find that reducing the damping timescale by an order of magnitude, while increasing wave yields an improved solution (Fig 5 bottom row). There are still errors as large as $1 \mathrm{~cm} / \mathrm{s}$; however they cover less of the domain. This result is unexpected by our linear theory presented in section 3 because with both parameter sets $\left(T_{0}=0.36\right.$ with $N=1200$ and $T_{0}=0.036$ with $\left.N=6000\right)$ the elastic wave is is likely due to the fact that reducing $T_{0}$ causes the elastic wave to travel faster and thus it can redistribute the stresses earlier during the pseudo timestepping procedure. Reducing $T_{0}$ also causes the elastic wave to be damped more rapidly during the pseudo timestepping. This is another potential reason that reducing 550 $T_{0}$ may result in a better approximate solution to the momentum equation. 
Next we reduce the advective timestep in order to satisfy the plastic wave CFL condition while maintaining the resolution of the elastic wave. With a 30 second timestep, 600 subcycles, and $T_{0}=0.36$, there are still errors in excess of 1 $\mathrm{cm} / \mathrm{s}$ (Fig 6 top row). Increasing the number of subcycles to 1200 and reducing ${ }_{555} T_{0}$ to 0.036 results in smaller error $(0.3 \mathrm{~cm} / \mathrm{s}$ in regions of high deformation $)$ (Fig 6 bottom row). We find that further reducing the advective timestep to 1 $\mathrm{s}$ with $T_{0}=0.036$ and 1200 subcycles effectively eliminates the error completely (not shown).

\subsubsection{EVP* Solver}

We test the EVP* method in the same 2-D idealized domain as described above. We use $\alpha=\beta$ as proposed by Kimmritz et al. [13] which as stated before implies that the damping time scale of the elastic wave is given by $T=0.5 \Delta t$ (i.e. $T_{0}=0.5$ ). With a 600 second advective timestep we use $\alpha=\beta=1000$ and 2000 subcycles in order to satisfy the stability condition given in Kimmritz et al. 13. With these numerical parameters, we find velocity errors greater that $1 \mathrm{~cm} / \mathrm{s}$ throughout the regions undergoing plastic deformation Fig 7 top row). The error pattern is largely similar to what was observed with the EVP method with $T_{0}=0.36$ and 1200 subcycles (Fig 6 top row). This is an indication that not resolving the plastic wave yields error in the EVP* method in a similar manner to that in the EVP method.

Reducing the advective timestep to 30 seconds effectively reduces the velocity error for the $\mathrm{EVP}^{*}$ method (Fig 7 bottom row). In this case we use $\alpha=\beta=500$ and 1200 subcycles in order to satisfy the stability condition given in Kimmritz et al. 13. Again the velocity error resembles the error found when using the EVP model with similar parameters i.e. $T_{0}=0.36$ and 1200 subcycles (Fig 6 top row). With the condition $\alpha=\beta$ we cannot reduce $T_{0}$ in order to achieve a more accurate approximate solution as we did for the EVP model. Perhaps allowing for $\alpha$ to be much less than $\beta$ one could achieve similar improvement, but we do not test this here. Note that in the simulations using the EVP* method we were unable to reduce the pseudo timestep derivative (1st term in 
Equ. 17) to machine precision at $10 \mathrm{~km}$ spatial resolution even when $\alpha$ and $\beta$ were set to 5000 , and $10^{6}$ subcycles were performed. In this case the magnitude of the residual vector reached $10^{-2}$, still orders of magnitude higher than the convergence criterion set for the JFNK method. At $80 \mathrm{~km}$ spatial resolution machine precision was reached (not shown). This result is in agreement with the results of Kimmritz et al. [13] who were unable to reach machine precision on 0.1 or 0.05 degree grids. This represents another source of model error in the $\mathrm{EVP}^{*}$ simulations presented here.

\section{Conclusions}

We use a linearized version of the Viscous-Plastic sea-ice model, and show that the plastic wave in this system of equations initially travels up to $26 \mathrm{~m} / \mathrm{s}$ for ice concentrations near $100 \%$. We present evidence that the noise noted in Hutchings et al. [15], Lipscomb et al. [17] and Lemieux et al. [16] arises from not resolving this plastic wave. We apply the CFL condition to this plastic wave and find, using numerical simulations, that failure to resolve the plastic wave results in first order error in 1-D deformation field when using the JFNK, EVP, or EVP* method. This error is effectively eliminated when using the Jacobian Free Newton Krylov method along with the IMplicit-EXplicit (IMEX) method previously proposed by Lemieux et al. [16. There is no analog to the IMEX method for the EVP or EVP* method, and therefore the model timestep must be small enough to satisfy the plastic wave CFL condition.

A second CFL condition is presented for the EVP model using the elastic wave speed as shown in Hunke and Dukowicz 7 ] and Hunke [8]. It is found that this elastic CFL condition is analogous to the stability condition derived by Kimmritz et al. 13 for the EVP* system of equations. We confirm that adhering to the appropriate stability condition for EVP or EVP* models is required to recreate the reference solution in 1-D. We find that the $\mathrm{EVP}^{*}$ model is slightly less sensitive to the resolution of the plastic wave in 1-D than the EVP model. 


\section{Acknowledgments}

James Williams is grateful to the North Slope Borough of Alaska for their financial support through the Eben Hopson Fellowship. James Williams and Bruno Tremblay are grateful for financial support from the Canadian Sea Ice and Snow Evolution (CanSISE) Network funded by NSERC Climate Change and Atmospheric Research program. Bruno Tremblay is also grateful for financial support from the NSERC Discovery program the Environment Canada Grants and Contribution program, and from the Office of Naval Research (N000141110977). 
We thank Sergey Danilov and an annonymous reviewer for their helpful comments which improved the clarity and content of this document.

\section{References}

1. Oliver J, Crary AP, Cotell R. Elastic waves in arctic pack ice. Transactions of the American Geophysical Union 1954;35.

2. Hunkins K. Seismic studies of sea ice. Journal of Geophysical Research $1960 ; 10$.

3. Pritchard RS. An elastic-plastic constitutive law for sea ice. Journal of Applied Mechanics 1975;.

4. Hibler WD. A dynamic thermodynamic sea ice model. Journal of Physical Oceanography 1979;9.

5. Gray JMNT. Loss of hyperbolicity and ill-posedness of the viscous-plastic sea ice rheology in uniaxial divergent flow. Journal of Physical Oceanography 1999;29.

6. Zhang J, Hibler W. On an efficient numerical method for modeling sea ice dynamics. Journal of Geophysical Research 1997;102.

7. Hunke EC, Dukowicz JK. An elastic-viscous-plastic model for sea ice dynamics. Journal of Physical Oceanography 1997;27.

8. Hunke EC. Viscousplastic sea ice dynamics with the EVP model: Linearization issues. Journal of Computational Physics 2001;170.

9. Lemieux JF, Tremblay B, Thomas S, Sedláček J. Using the preconditioned Generalized Minimum RESidual (GMRES) method to solve the sea-ice momentum equation. Journal of Geophysical Research 2008;113.

10. Lemieux JF, Tremblay B, Sedláček J, Tupper P, Thomas S, Huard D, Auclair JP. Improving the numerical convergence of viscous-plastic sea ice 
models with the jacobian-free newton-krylov method. Journal of Computational Physics 2010;229.

11. Lemieux JF, Knoll DA, Tremblay B, Holland DM, Losch M. A comparison of the Jacobian-Free NewtonKrylov method and the EVP model for solving the sea ice momentum equation with a viscous-plastic formulation: A serial algorithm study. Journal of Computational Physics 2012;231.

12. Bouillon S, Fichefet T, Legat V, Madec G. The elastic-viscous-plastic method revisited. Ocean Modelling 2013;71.

13. Kimmritz M, Danilov S, Losch M. On the convergence of the modified Elastic-Viscous-Plastic method for solving the sea ice momentum equation. Journal of Computational Physics 2015;296.

14. Kimmritz M, Danilov S, Losch M. The adaptive EVP method for solving the sea ice momentum equation. Ocean Modelling 2016;101.

15. Hutchings JK, Jasak H, Laxon SW. A strength implicit correction scheme for the viscous-plastic sea ice model. Ocean Modelling 2004;7.

16. Lemieux JF, Knoll DA, Losch M, Girard C. A second-order accurate in time IMplicit-EXplicit (IMEX) integration scheme for sea-ice dynamics. Journal of Computational Physics 2014;263.

17. Lipscomb W, Hunke E, Maslowski W, Jakacki J. Ridging, strength, and stability in high-resolution sea ice models. Journal of Geophysical Research: Oceans 2007;112.

18. McPhee MG. Sea ice drag laws and simple boundary layer concepts including application to rapid melting. Tech. Rep. 82-4; Cold Regions Research and Engineering Laboratory; Hanover, N.H; 1982.

19. Lemieux JF, Tremblay B. Numerical convergence of viscous-plastic sea ice models. Journal of Geophysical Research 2009;114. 
20. Hibler WD, Ip CF. The effect of sea ice rheology on arctic buoy drift. Ice Mechanics, American Society of Mechanical Engineers 1995;204.

21. Hopkins MA. Four stages of pressure ridging. Journal of Geophysical Research 1998;103.

22. Weeks WF, Assur A. The mechanical properties of sea ice. Tech. Rep. II-C3; Cold Regions Research and Engineering Laboratory; Hanover, N.H.; 1967.

23. Lindsay RW, Zhang J, Rothrock DA. Sea ice deformation rates from satellite measurements and in a model. Atmosphere-Ocean 2003;41.

24. Lindsay RW, Stern HL. The RADARSAT geophysical processor system: Quality of sea ice trajectory and deformation estimates. Journal of Atmospheric and Oceanic Technology 2003;20.

25. Marsan D, Stern H, Lindsay R, Weiss J. Scale dependence and localization of the deformation of artic sea ice. Physical Review Letters 2004;93. 


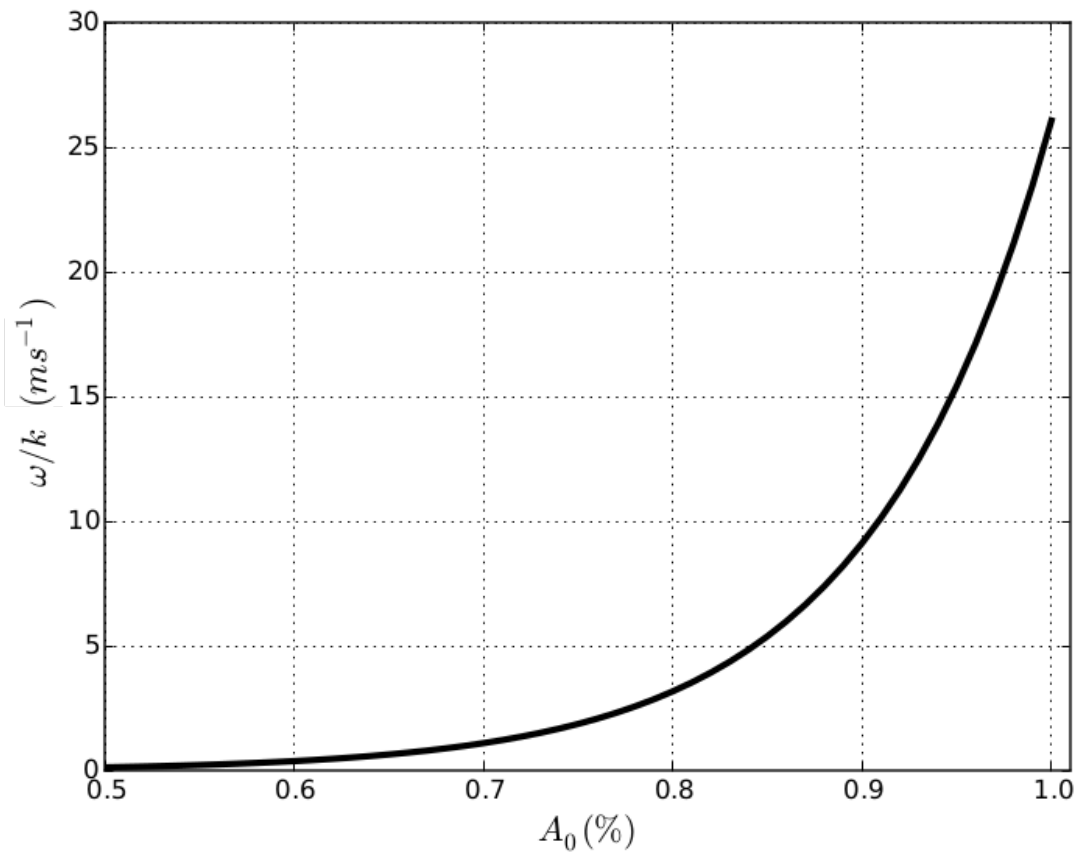

Figure 1: Plastic wave speed for the linearized set of governing equations as a function of the base state concentration field, $A_{0}$. Note that the plastic wave speed does not depend on the base state thickness field. 

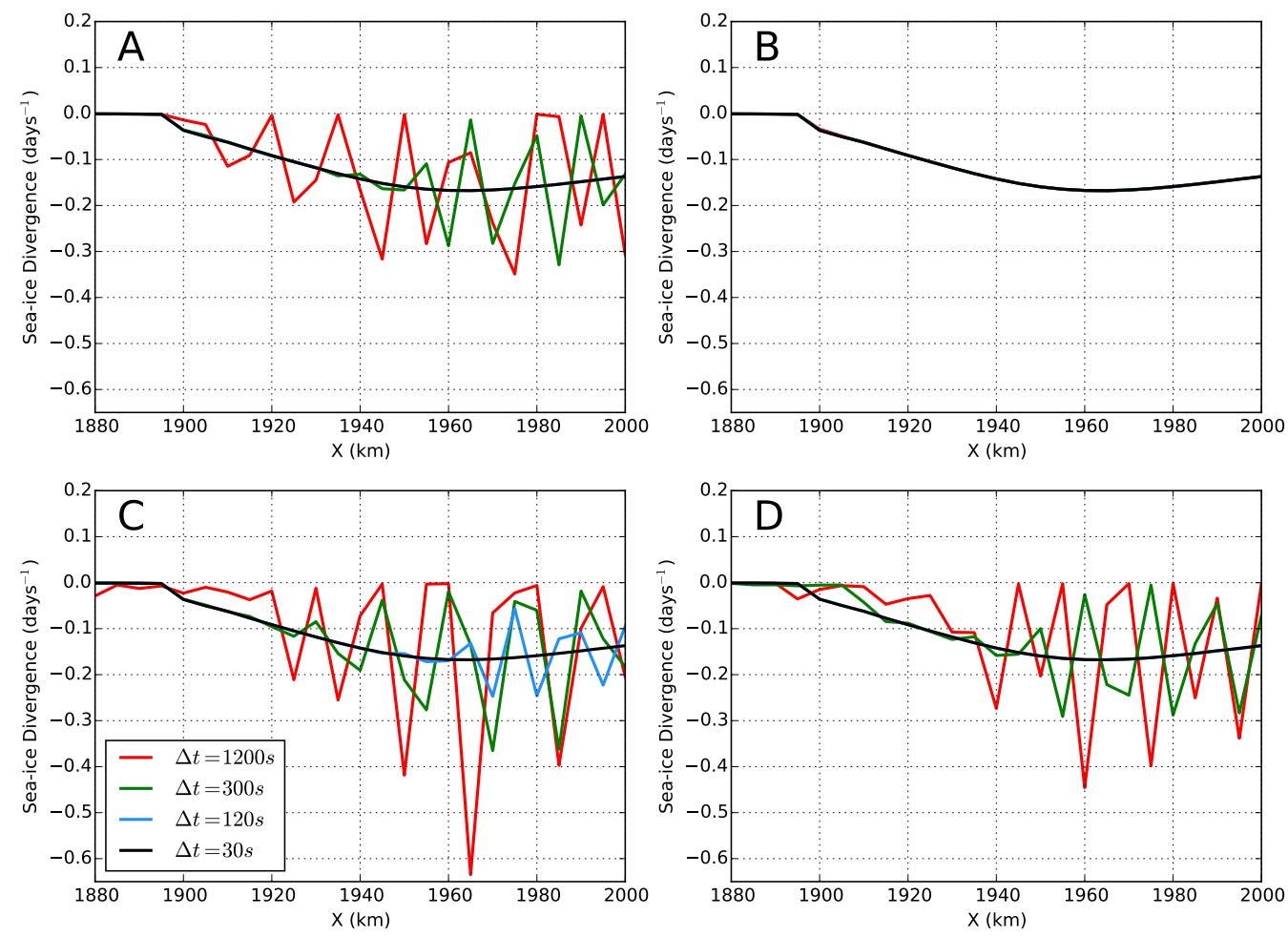

Figure 2: Sea-ice divergence at time $=24 \mathrm{hrs}$ for the A) JFNK, B) JFNK+IMEX, C) EVP with 1200 subcycles, D) EVP* with 4000 subcycles. In all panels the color of the curve indicates the advective timestep: black: $\Delta t=30 \mathrm{~s}$, blue: $\Delta t=120 \mathrm{~s}$, green: $\Delta t=300 \mathrm{~s}$, and red: $\Delta t=1200 \mathrm{~s}$. In some panels the curves lie directly on top of eachother. The spatial resolution of all simulations is $5 \mathrm{~km}$ and $\alpha=\beta=2000$. 


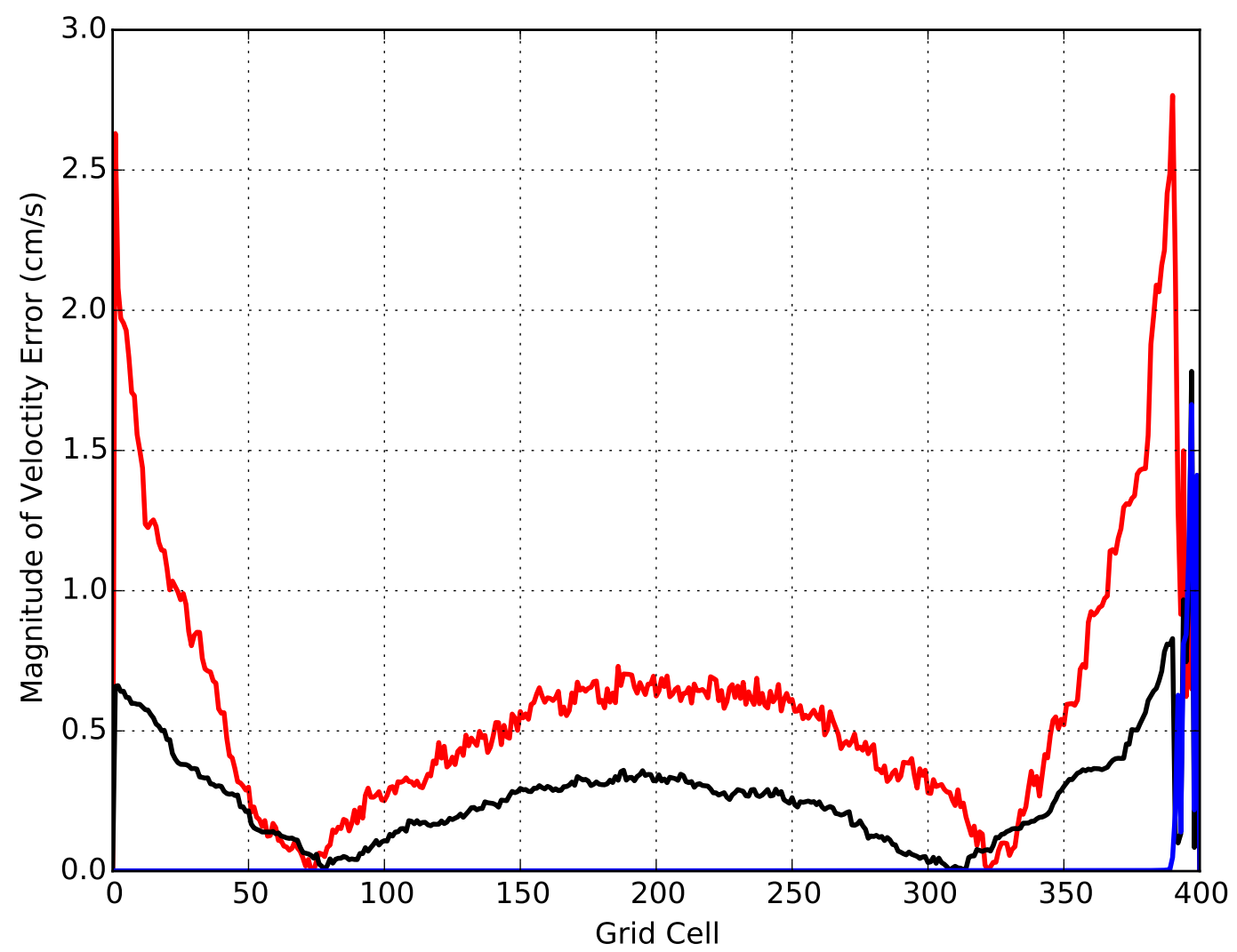

Figure 3: Errors in the EVP simulated sea-ice velocity field at time $=6$ hrs with a 600 sec timestep for various numbers of subcycles: 430 (red), 860 (black) and 1720 (blue). 860 subcycles is the CFL criterion to resolve the EVP elastic wave (see Equ 49 . 

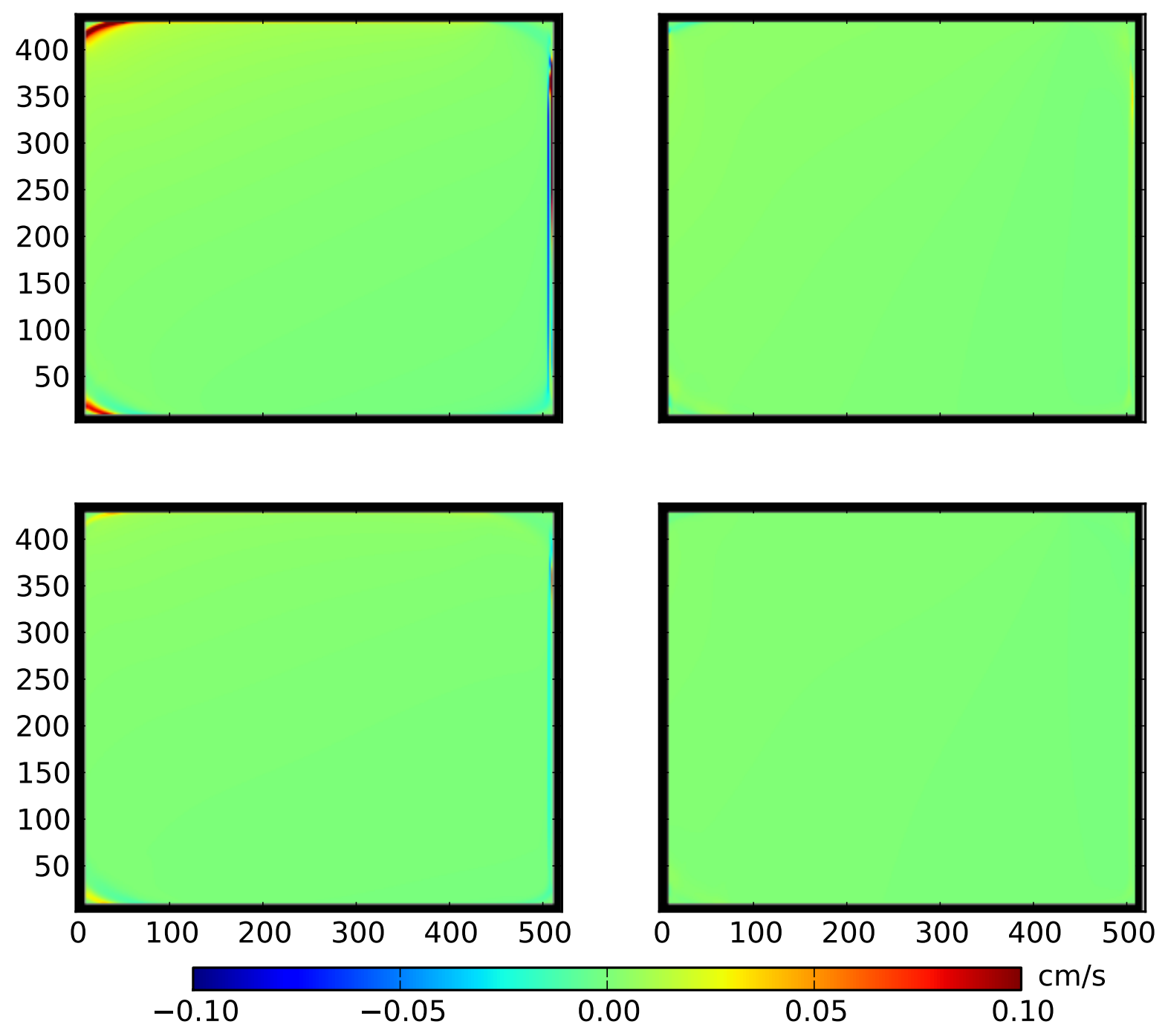

Figure 4: Error of the x-component of velocity (left column) and y-component of velocity (right column) at time $=6 \mathrm{hrs}$, simulated by the JFNK model without IMEX (top row) and with IMEX (bottom row). The scale of the color bar is capped to $\pm 0.1 \mathrm{~cm} / \mathrm{s}$. 

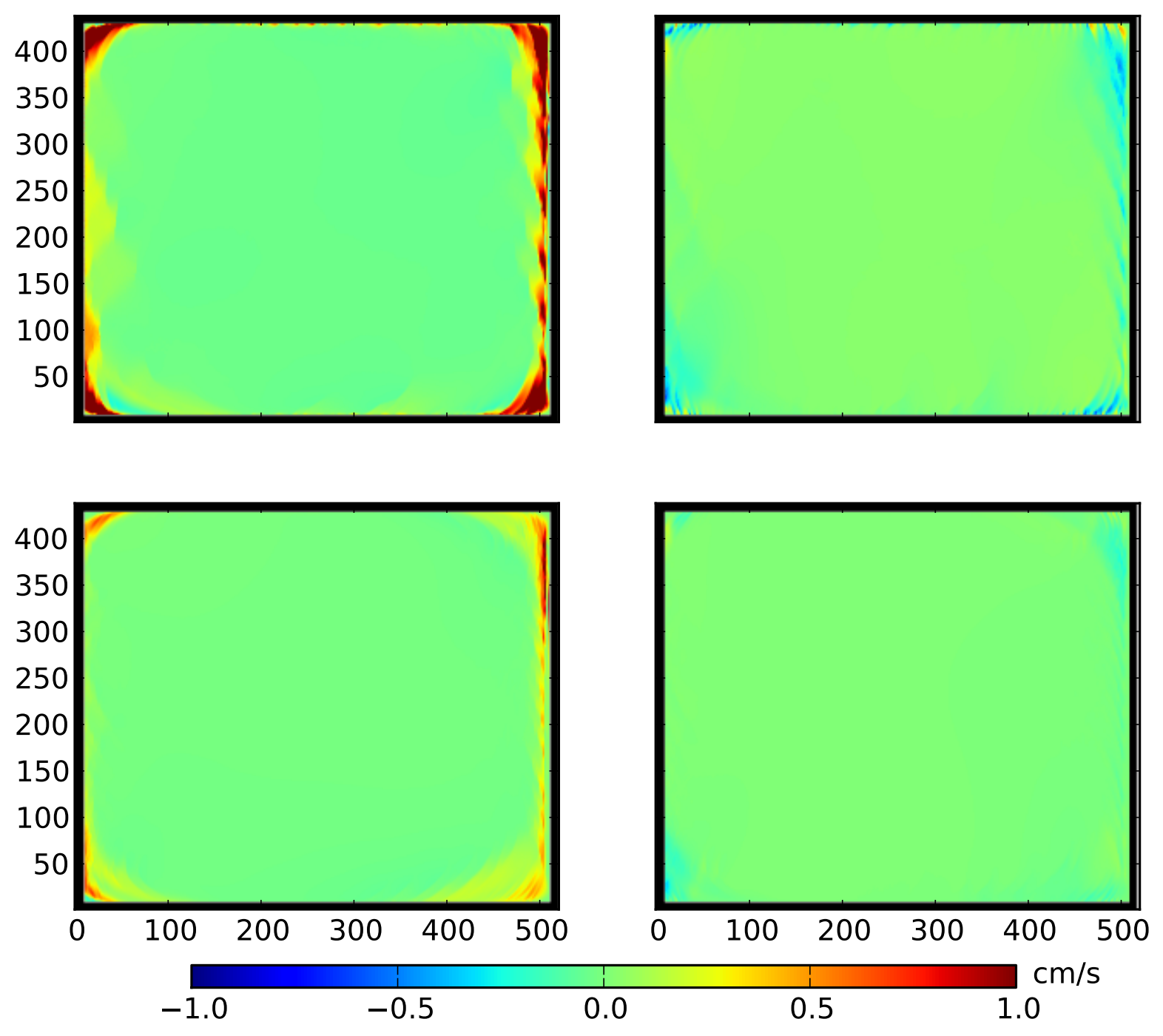

Figure 5: Error of the x-component of velocity (left column) and y-component of velocity (right column) at time $=6 \mathrm{hrs}$, simulated by the EVP model with 1200 subcycles and $T_{0}=$ 0.36 (top row) and 6000 subcycles and $T_{0}=0.036$ (bottom row). The scale of the color bar is capped to $\pm 1 \mathrm{~cm} / \mathrm{s}$. 

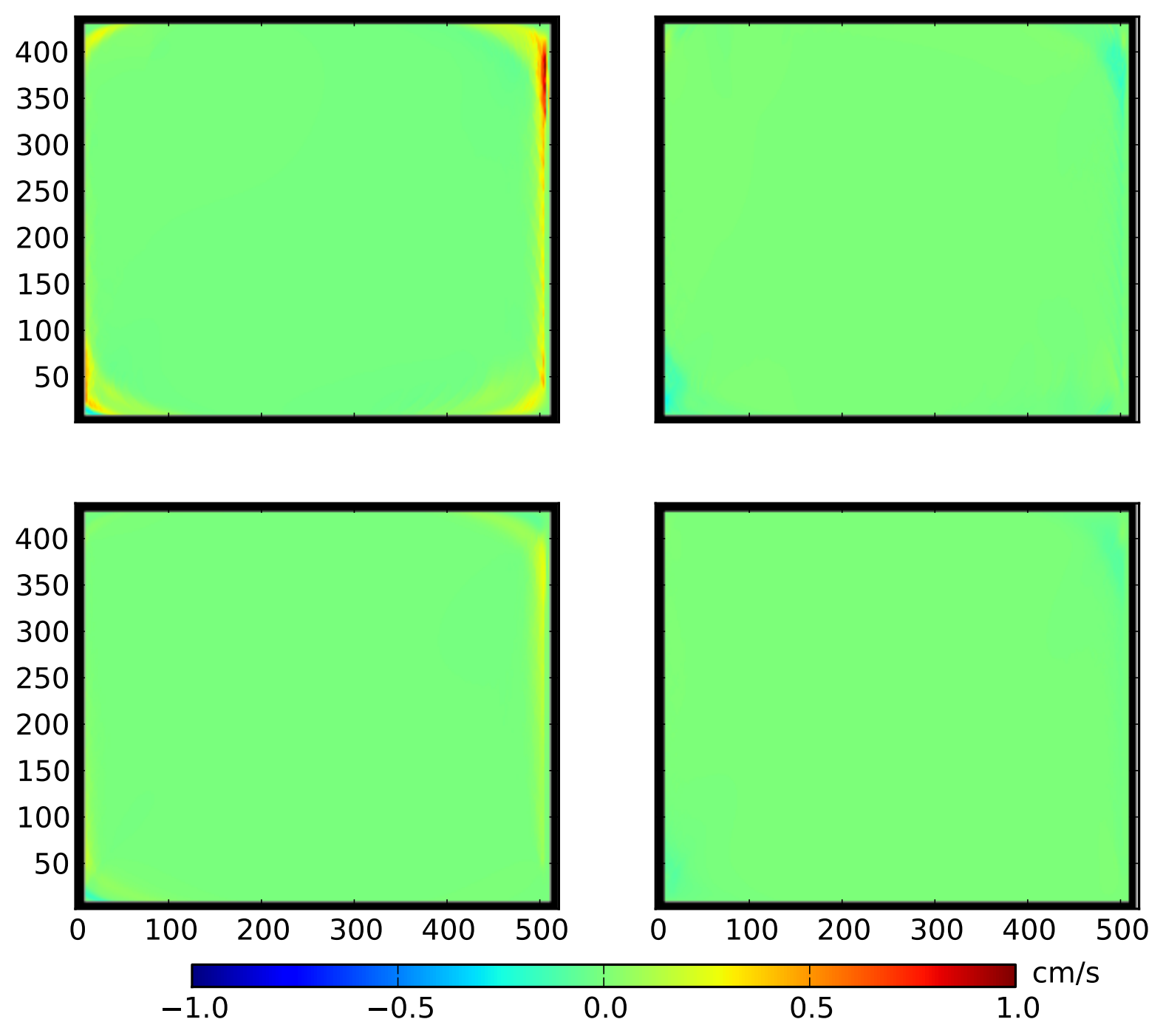

Figure 6: Error of the x-component of velocity (left column) and y-component of velocity (right column) at time $=6 \mathrm{hrs}$, simulated by the EVP model with $\Delta t=30 \mathrm{~s}$ with 600 subcycles and $T_{0}=0.36$ (top row), and 1200 subcycles and $T_{0}=0.036$ (bottom row). The scale of the color bar is capped to $\pm 1 \mathrm{~cm} / \mathrm{s}$. 

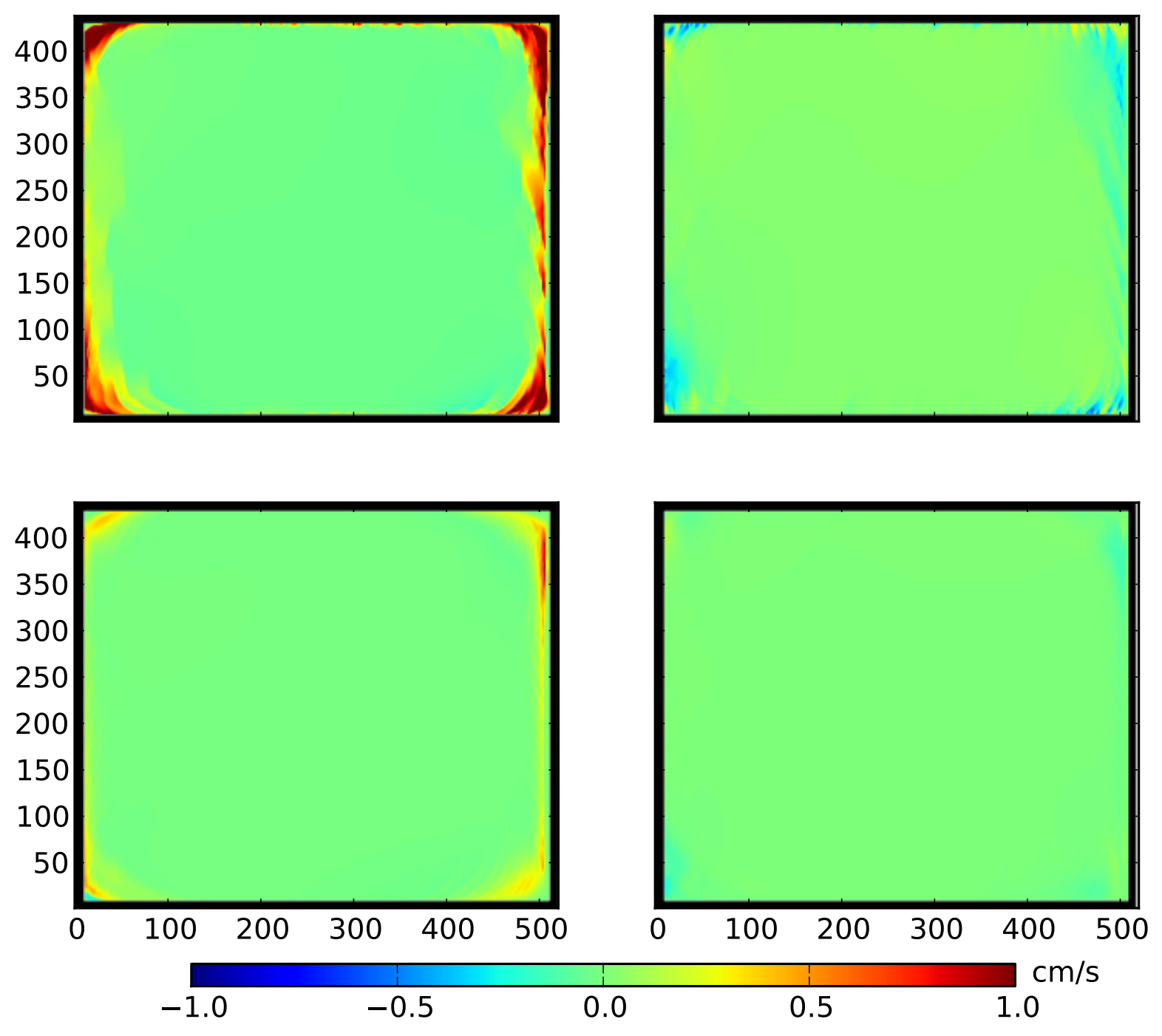

Figure 7: Error of the x-component of velocity (left column) and y-component of velocity (right column) at time $=6 \mathrm{hrs}$, simulated by the EVP ${ }^{*}$ model with $\Delta t=600 \mathrm{~s}, \alpha=\beta=1000$, 2000 subcycles (top row), and $\Delta t=30 s, \alpha=\beta=500,1200$ subcycles (bottom row). The scale of the color bar is capped to $\pm 1 \mathrm{~cm} / \mathrm{s}$. 
Table 1: Definition and typical values of constants and parameters used in the viscous-plastic sea-ice model

\begin{tabular}{ccc}
\hline \hline Symbol & Definition & Typical Value \\
\hline$\rho_{i}$ & Density of sea ice & $900 \mathrm{~kg} \mathrm{~m}^{-3}$ \\
$\rho_{a}$ & Density of air & $1.3 \mathrm{~kg} \mathrm{~m}^{-3}$ \\
$\rho_{w}$ & Density of sea water & $1026 \mathrm{~kg} \mathrm{~m}^{-3}$ \\
$C_{d a}$ & Air drag coefficient & $1.2 \times 10^{-3}$ \\
$C_{d w}$ & Water drag coefficient & $5.5 \times 10^{-3}$ \\
$\theta_{d a}$ & Air drag turning angle & $25^{\circ}$ \\
$\theta_{d w}$ & Water drag turning angle & $25^{\circ}$ \\
$f$ & Arctic Coriolis parameter & $1.46 \times 10^{-4} \mathrm{~s}^{-1}$ \\
$\mathrm{P}^{*}$ & Ice strength parameter & $27.5 \times 10^{3} \mathrm{~N} \mathrm{~m}^{-2}$ \\
$\mathrm{C}$ & Ice concentration parameter & 20 \\
$\mathrm{e}$ & Ellipticity of the yield curve & 2 \\
$\mathrm{~g}$ & Gravitational acceleration & $9.81 \mathrm{~ms}^{-2}$ \\
\hline
\end{tabular}

